\title{
Laboratory Studies of Stratified Convection with Multiple States
}

\author{
J. A. Whitehead \\ Department of Physical Oceanography \\ MS\# 21, Woods Hole Oceanographic Institution \\ Woods Hole, Massachusetts 02543 \\ jwhitehead@whoi.edu \\ Keith Bradley \\ Department of Physical Oceanography \\ MS\# 2, Woods Hole Oceanographic Institution \\ Woods Hole, Massachusetts 02543 \\ kbradley@whoi.edu
}

13 January 2005

\begin{abstract}
Keywords: Mixing, ocean convection, salt balance, water circulation, ocean circulation, water masses
\end{abstract}

\begin{abstract}
A simplified box model of the cooling of a salt-stratified ocean has been constructed in the laboratory to test a theory that predicts multiple equilibria if certain conditions exist. An isothermal basin of water had a thin layer of fresh water over salt water. Beside this was a smaller basin connected to the large basin by horizontal tubes at the top, middle and bottom. The small basin was cooled from above. If the top tube has more flow resistance than the bottom tubes, theory indicates that as cooling temperature $T^{*}$ is made colder, there is a sudden transition between two flow states. The velocities in the tubes jump to greater values, while salinity and temperature in the small basin jump to another value. These multiple states are found in the laboratory experiments along with some states that oscillate. Laboratory measurements and layered model calculations for hysteresis and the jump of temperature and salinity agree qualitatively, but there is only rough quantitative agreement.
\end{abstract}




\section{Introduction}

Only a small percentage of the ocean surface experiences a deep mixed layer that leads to sinking of surface water in the deep ocean during wintertime cooling (Stommel, 1962). The known localities of deep convection in the middle of winter are restricted to the Greenland-Norwegian Sea, the Labrador Sea (Lab Sea Group, 1998), the Medoc regions in the Mediterranean (MEDOC group 1969), the Weddell Sea (Gordon, 1991), and the Ross Sea, although there may be some additional regions (R. Pickart pri. Comm.). Using area estimates from maps and from Table 4 in "the Oceans" (Sverdrup, Johnson and Fleming 1942), each area is less than $2000 \mathrm{~km}$ on a side, so their combined total area is less than $7 \%$ of the area of the ocean of $3.2 \times 10^{8} \mathrm{~km}^{2}$. Therefore, to a first approximation, much of the ocean's water below $2000 \mathrm{~m}$ entered the deep ocean through these small areas.

The smallness of this area is partly due to a low salinity layer near the ocean surface that is found virtually everywhere in polar regions. Either this layer must be substantially thinned by surface flow divergence, or an increase of salinity of this fresher surface water is required for the mixed layer to deepen to depths greater than about 500 m. Without such an increase, a polar ocean accumulates surface ice before the surface water becomes dense enough to sink to great depths. For example, in the Arctic Ocean, although the ice thickens in winter and this causes surface salinities to increase, the low salinity layer never vanishes and therefore no water sinks from near the surface to great depths.

The transition from a convective flow that involves a slow flow driven by salinity differences to a rapid flow driven by temperature differences is very common in box models of ocean circulation when both temperature and salinity are included in the equation that expresses convection. Marotzke (1994), Whitehead (1995), Hearn and Sidhu (1999), and Rahmstorf and Ganopolski (1999), summarize a variety of studies for example. Observations of jumps of flow configurations from one distinct flow state to another are also found in numerical ocean circulation models (Bryan 1986, Manabe and Stouffer 1995).

Obviously box models and ocean circulation climate numerical models involve many simplifications. It is possible that many real situations, which have more degrees of 
freedom, do not exhibit the clear jumps, multiple equilibria and hysteresis found in the box and numerical models. Indeed, at present, no such large jumps between two distinct flow states have been observed in nature, either in the deep ocean or in smaller basins that are supplied by freshwater such as shallow seas, shelves, fjords or estuaries. This has stimulated our present laboratory program to investigate nonlinear multiple equilibria in actual temperature-salinity driven flows.

In an experiment that attempts to mimic the classic Stommel (1961) box model, a chamber was heated from below and exposed to a steady flux of salt water distributed along the top surface (Whitehead 1996, 1998). Two locally stable modes of circulation were found within a well-documented range of the temperature and salinity forcing; one mode is primarily temperature driven (the T-mode) and one mode is primarily salinity driven (the S-mode). Hysteresis and large jumps in temperature and salinity are found as the system migrates back and forth between the two modes. This experiment had vigorous mixing within the chamber from the combined effects of heating from below and a uniform saltwater flux from the top. Therefore, it is strongly constrained to be very similar to the Stommel model, and the good agreement of experiment and theory is not surprising.

Next, laboratory experiments (Whitehead et al. 2003) were conducted to explore the consequences of more limited mixing. The mixing was limited by introducing saltwater flux through a confined source held above the bottom within the chamber that was also heated from below. The salt water descended as a turbulent plume, so that plume theory was used to estimate the magnitude of mixing. In addition, a narrow vertical slot in the sidewall between the chamber and the reservoir allowed partially mixed salt water to leave the test chamber through the slot and to be replaced by isothermal fresh water flowing in though the slot from the reservoir. The result was a three-layer structure for the S-mode, which corresponds to shallow convection, and a two-layer structure for the T-mode, which corresponds to deep ocean convection. Hysteresis in the experiments with limited mixing is considerably smaller than hysteresis in the fully mixed box model theory.

We report here results of new laboratory experiments inspired by a new theory of layered convection. This theory shows hysteresis and jumps as cooling temperature is 
changed (Whitehead 2000), but it differs from the T-S models cited above because the ambient fluid is layered. Its development was motivated by the fact that the layer of low salinity in the polar regions of the earth was not clearly represented in the older boxmodel studies. Section 2 reviews this theory and its predictions. Section 3 describes the laboratory experiments and the results. Section 4 describes the experimental results, including a new oscillation in the experiment. Section 5 discusses some aspects of the results and section 6 summarizes them.

\section{Review of Theory}

The configuration for the theory (and subsequent experiments) consists of two basins as shown in Figure 1. The small basin on the left is subjected to constant cold temperature at the top, which cools the top of the small basin at a specified rate. This basin is connected via three horizontal tubes to a large isothermal basin of salt water on the right covered by a shallow layer of fresh water. Temperature and salinity of the large basin are forever fixed, so the large basin on the right provides boundary conditions at the end of each tube. The tubes were adopted to identify the effects of deep, intermediate, and shallow flows into and out of the small basin. This model produces multiple equilibria as will be shown below. The middle tube in Figure 1 is important in this mechanism because entrainment of deep salt water into the small basin is activated if salt water flows into that layer through the middle tube.

\section{Cold bath}

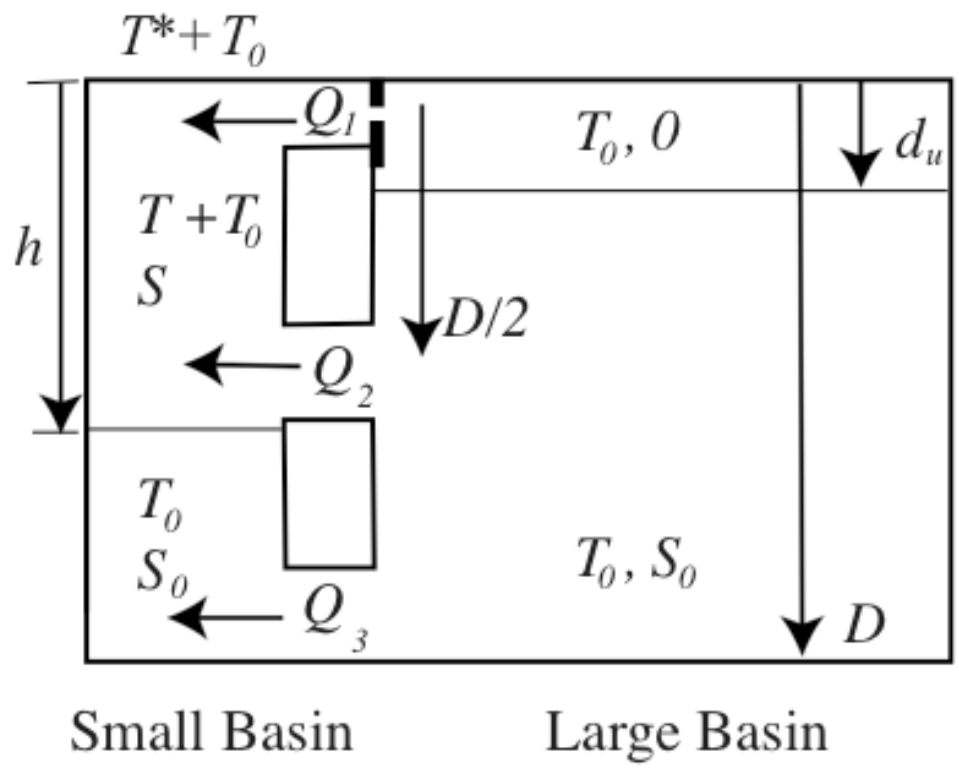


Figure 1. A large region with a layer of fresh water above salty water lies next to a small basin cooled from above. Three tubes connect the basins at top, middle, and bottom. The layer in the small basin has depth $h$.

Figure 1 shows many relevant parameters. In addition to these, a flow law through the tubes

$$
Q_{i}=K_{i} \Delta p_{i}
$$

is required where $K_{i}$ is the resistance coefficient and $\Delta p_{i}$ is the pressure drop across each tube for $i=1,2,3$. These all result in four dimensionless numbers for the dynamics of this model. The first one measures the strength of cooling, and is linearly proportional to the rate of cooling itself. It is the dimensionless number

$$
\tilde{T}^{*}=\alpha T^{*} / \beta S_{0},
$$

where $\alpha$ is the coefficient of thermal expansion and $\beta S_{0}$ is the relative change in density between the fresh water layer and the salty water below (Figure 1 shows the variables). The temperature of a cooling bath is $T^{*}+T_{0}$, where $T_{0}$ is temperature of the fluid in the large basin on the left, and $T *$ is the negative value of temperature added to the top of the small basin. Heat transfer rate at the top of the small basin is

$$
H=K_{T}\left(T^{*}-T\right)
$$

and since both temperatures are normally negative this is negative and there is a net cooling of the fluid below it. In the experiment, this cooling produces cellular convection in the fresh water layer that keeps it mixed up. The constant $K_{T}$ is conductivity and the dimensionless equivalent has a value of about one for this experiment. It was set to a value of one in the theory. Since time scale in the theory is defined by the exponential time for a fresh water layer to fill the small basin starting from $h=0$, the dimensionless conductivity parameter being one means that fresh water flowing into the small basin will have a noticeable change in temperature from surface cooling. For dynamical reasons, the top tube is taken to have a different resistance than the bottom two tubes. This increased resistance is sketched as a plate with a small hole in figure 1 . The third dimensionless number

$$
\gamma=K_{1} / K_{2}
$$


is a measure of the ratio of the flow resistance of the top tube to the flow resistance of the bottom two tubes. We do not specify what fluid mechanics governs flow resistance, it could be either turbulent or laminar pipe flow. However, if flow through the tubes is governed by laminar Stokes flow, $\gamma$ is proportional to the ratio of tube radii to the fourth power for tubes of the same length. The fourth dimensionless number is depth of the top layer compared to the vertical tube spacing

$$
\tilde{d}=d_{u} / D
$$

- Multiple equilibria are found over a wider range of $\gamma$ for smaller $\tilde{d}$.

The results as a function of $\tilde{T}^{*}$ (always negative) for one value of $\gamma, d^{\prime}$ are shown in Figure 2a (from Whitehead 2000). Dimensionless salinity is $\tilde{S}=S / S_{0}$ and dimensionless temperature (again, always negative) is $\tilde{T}=\alpha T / \beta S_{0}$. For slightly negative $\tilde{T}^{*}$ (toward the right in this figure) lies the fresh-mode. Fresh water flows into the small chamber in the top tube where it becomes cooled, denser, and flows out through the middle tube. For more negative $\tilde{T}^{*}$ i.e. greater cooling, the cold freshwater layer extends to the bottom of the small basin so the water leaves through both the middle and the bottom tubes. This is evident by a change in slope of the lower line for temperature and density. The corresponding salinity is zero for both of these. If the cooling is even stronger, a transition to the mixed-mode takes place at the large jump shown in Figure 2a. The flow in the middle tube reverses direction to produce this transition. Salty water enters the small basin through the middle tube, but it is lighter than the very cold mixture in the small basin. As the warm salt-water plume rises to the top of the small basin, it cools and changes salinity by mixing with fluid already in the small basin. At the top of the small basin the mixture becomes very cold by conduction of heat out of the top boundary, and then it sinks to the bottom and leaves the small basin through the bottom tube. 

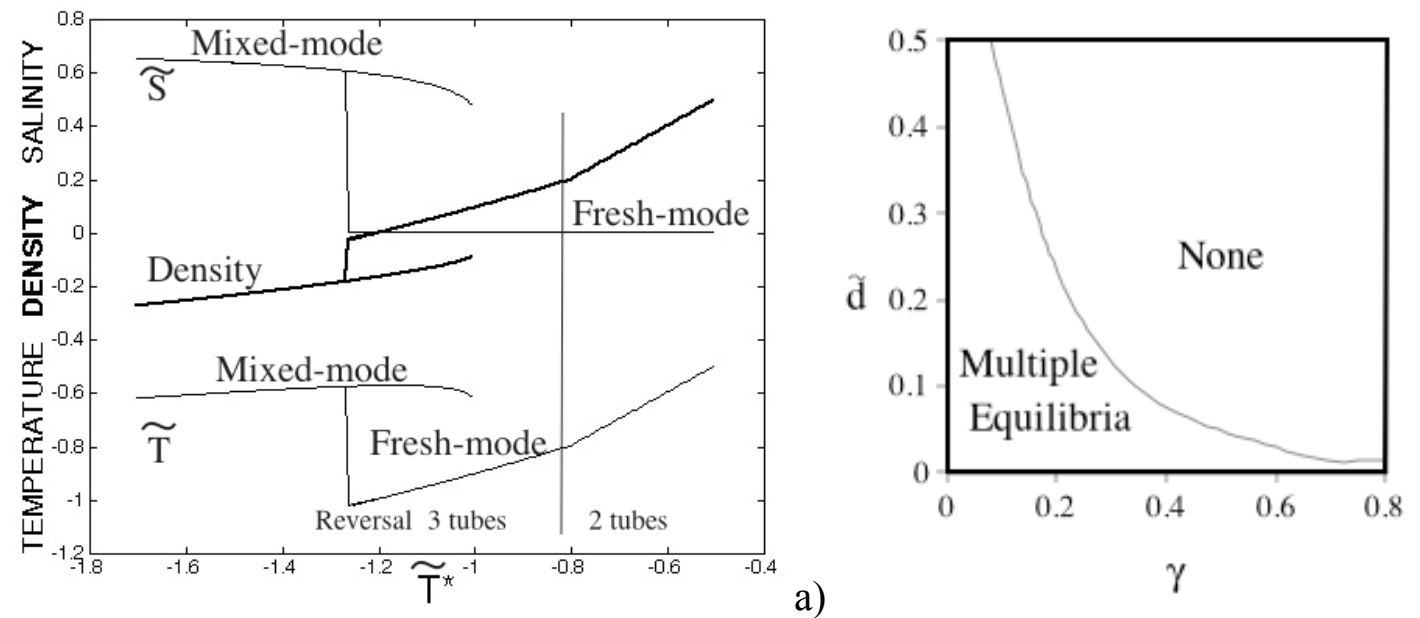

a)

b)

Figure 2. a) Results of the numerical calculations for $\gamma=d^{g}=0.05$.

Dimensionless temperature is on the bottom, salinity on the top, and density is the double thick curve. b) The region in parameter space where multiple equilibria are found. The parameter $\tilde{d}$ is ratio of layer depth to distance between top and bottom tubes with the middle tube halfway. The parameter $\gamma$ is ratio of volume flux through the top tube to either other tube for fixed pressure drop across both tubes.

If a jump from the fresh mode to the mixed mode occurs, there is a range of $\tilde{T}^{*}$ that has two stable flows for each value of $\tilde{T} *$. Having two stable flows is also described as having multiple equilibria. This range of $\tilde{T}^{*}$ implies that if one were to conduct an experiment by slowly making $\tilde{T}^{*}$ more negative, then the flow would stay in the fresh-mode until the point in figure 2 labeled "reversal" is encountered. At that point, the flow jumps to the mixed mode and stays in that mode as $\tilde{T}^{*}$ is made more negative. Later, if $\tilde{T}^{*}$ is slowly made less negative, the jump back to the fresh-mode would not be found at the first reversal value of $\tilde{T}^{*}$, but instead the jump would occur at the far righthand end of the mixed mode curve. In this case, we would say that hysteresis (or history dependence) has taken place, since the jumps were determined by history of the value of $\tilde{T} *$.

Not all values of $\gamma, d^{\prime}$ produce jumps and hysteresis. Figure $2 \mathrm{~b}$ shows calculations (Whitehead 2000) of the range of $\gamma, d^{\prime}$ with jumps. They exist only for $\gamma<1$ in which case the top tube has more flow resistance than either bottom tube. Figure 2 
also shows that jumps are found over a wider range of $\gamma$ with a shallower upper layer in the large basin.

\section{The Experiment}

\subsection{Apparatus}

The laboratory apparatus described in this section was directly motivated by the theory reviewed in Section 2 and the layout sketched in figure 1. A photograph is shown in figure 3. The large basin consisted of an acrylic basin with $20 \mathrm{~cm}$ sides that have $1 \mathrm{~cm}$ thick walls for good thermal insulation. This was connected via three vertically aligned horizontal tubes to a small basin $20 \mathrm{~cm}$ high. The small basin had the form of a vertical cylinder with a diameter of $5.1 \mathrm{~cm}$ and $1 / 2 \mathrm{~cm}$ wall thickness to improve thermal insulation. The vertical distance between the centers of the adjacent tubes was $9 \mathrm{~cm}$. Radius of the three tubes was $0.95 \mathrm{~cm}$. The top tube had a plate $1.1 \times 10^{-3} \mathrm{~m}$ thick cementer across it at the large basin end. The plate had a $3 \times 10^{-3} \mathrm{~m}$ diameter hole to increase flow resistance.

Control baths accurate to $\pm 0.2^{0} \mathrm{C}$ supplied water to the experiments. The temperature of the large basin was the same as the cold external bath within $\pm 0.5^{\circ} \mathrm{C}$ in all cases. In all runs, the hot and cold baths were set with hot and cold temperatures centered above and below the approximate room temperature of $20{ }^{\circ} \mathrm{C}$ to minimize heat loss to the room. Fresh water at fixed temperature $T_{o}$ was pumped into the top of the large basin at a rate of about $0.1 \mathrm{l} / \mathrm{min}$., and salt water of the same temperature was pumped into the bottom of the large basin at the same rate. The same total flux of water was removed through an exit tube that was held at a fixed elevation above the bottom of the basin. This maintained a sharp interface between the fresh and the salty water at depth $d_{u}$. In figure 3 , the layer is dyed and thus is visible. The fresh water in the top was frequently injected with dye so the fresh water could be easily seen. A heat exchanger supplied with water of fixed temperature $T_{0}$ was immersed in the large basin along the side opposite the tubes. The large basin temperature was monitored routinely and it stayed at $T_{0}$ with deviation at all locations less than $\pm 0.5^{\circ} \mathrm{C}$. 
The cooler consisted of a copper cylindrical cooling chamber whose horizontal surface was in contact with the water surface in the small basin. Temperature was $T^{*}+T_{0}$. A small gap existed between the cap and the inner wall of the small basin so the water surface remained at atmospheric pressure. Although it would be better in a quantitative sense to provide extensive thermal insulation around this apparatus, the apparatus in figure 3 provided us with the opportunity to visually see the dyed fresh water and to observe the flow directions everywhere with injected dye. The thick acrylic walls provided partial thermal sidewall insulation, but still we estimate up to $70 \%$ heat lost to the room.

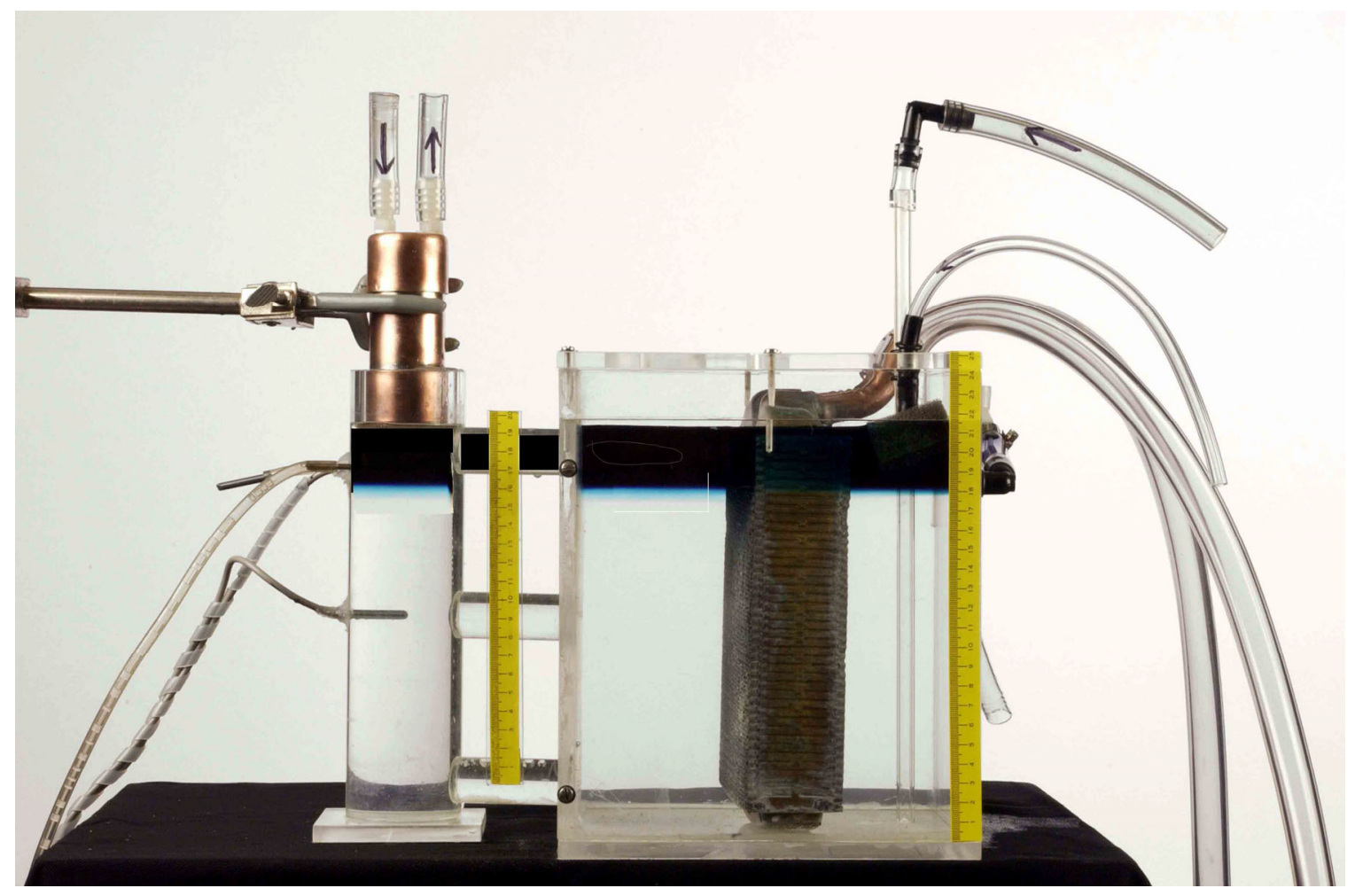

Figure 3. Elevation-view photograph of the beginning of an experiment. Three tubes connect the small basin on the left to the large basin on the right. The fresh water at the top of the large basins is dyed and the salt water below it is clear. The copper cooler touches the top of the water in the small basin. One tube brings fresh water into the top of the large basin and another pump brings salt water into the bottom. Water flows out at the level of the interface through a drain at depth $d_{u}$ on the right. In addition, a small automobile radiator keeps the water in the large basin within $\pm 0.5^{\circ} \mathrm{C}$ of the desired large basin temperature. Two temperature probes are shown to the left at $2.54 \mathrm{~cm}$ and at $10 \mathrm{~cm}$ below the top surface in the 
small basin. A tube is also visible at the level of the top probe that pumps a sample of water to the densiometer. Another tube to return the water to the experiment lies behind this tube.

\subsection{Procedure}

To prepare the water, large storage containers were filled with fresh water with a density of $998.2 \mathrm{kgm}^{-3}$ and others were filled with a mixture of fresh and salt water with a density of $1003.4 \mathrm{kgm}^{-3}$ at $20^{\circ} \mathrm{C}$. This gave a density difference between the two layers in the reservoir of $5.2 \mathrm{kgm}^{-2}$. This makes the salinity scale $\beta S_{0}=0.0052$.

An experiment was started by filling both basins with the fresh-water layer above the salt-water layer. After this, fresh and salt-water pumps were switched on so that the waters were continually renewed in the large basin. Also, the temperatures of the control baths were set at the desired values. Then, the system was left to come to equilibrium. This typically took well over one hour. Data were subsequently recorded until the run ended. Temperatures $T^{*}$ and $T_{0}$ were systematically varied. To minimize heat loss to the outside, the average temperature was kept close to room temperature by making $\left(T^{*}+T_{0}\right) / 2=20^{\circ} C$.

Data in the final experiments included temperature and density measurements. Precision for temperature was $\pm 0.05^{\circ} \mathrm{C}$ and for density was $\pm 0.05 \mathrm{~kg} / \mathrm{m}$. Every $15 \mathrm{~s}$, the temperature in the small basin was automatically measured with two probes. One probe was $2.5 \mathrm{~cm}$ below the top of the small basin. The other probe was $10.0 \mathrm{~cm}$ below the top of the small basin. In addition the density of a sample was found using a densiometer to determine density at $20^{\circ} \mathrm{C}$. Water was also steadily withdrawn at the depth of $2.5 \mathrm{~cm}$ below the top of the small basin. It was pumped into a densiometer at a rate that was slow enough to allow the sample to reach $20^{\circ} \mathrm{C}$ there. The water was then returned to the small basin.

All experiments were also continuously monitored with a shadowgraph of the small basin recorded by time-lapse videos. Not only did these reveal the location of interfaces in the small basin, but also they could record flow direction and a crude measure of velocity speed with hand injected dye at various times and locations. 


\section{Experimental Results}

\subsection{Qualitative observations}

Four different flow regimes were found, both with quantitative data, which are described in the next section, and by reviewing the video data, as described in this section. Three regimes have steady flow and the fourth is cyclic with periods greater than one hour.

Regime number 1 is sketched in figure $4 \mathrm{a}$. It has exchange flow in the middle tube, and is therefore not in the theory encompassed by Whitehead (2000), which assumed unidirectional tube flow. The interface in the small basin is located below the mid-plane of the middle tube, and there is an interface between dyed and clear fluid within the middle tube. Injected dye clearly showed that water flows out of the small basin along the top of the middle tube, and water below the interface in the middle tube flows into the small basin along the bottom of this tube. If the interface lay below the middle tube, so that the dimensionless layer depth in the small basin $\tilde{h}>1 / 2$, then the clear salt water entering the small basin sinks through the fresh water as a turbulent plume. The mixed water penetrates down through the interface in the small basin and occupies the region below the interface. This mixed water is darker than the clear water in the large tank, so we infer that salinity of the deep water of the small basin is lower than the salinity of deep water in the large basin. Since the interface level in the small basin is stationary even though this volume of water is continually supplied, water must also leave the small basin through the bottom tube. This was verified with injected dye.

$\underline{\text { Regime number } 2}$ has a steady flow sketched in figure $4 \mathrm{~b}$. This regime corresponds most closely to the regime called the "fresh-mode" in section 2 and in figure 2. This mode is indicated by the presence of only dyed water in the middle tube flowing out of small basin. Clear water is completely absent at the bottom of the middle tube. The interface has reached the bottom of the small basin in this regime and dyed freshwater flows out of the small basin through the bottom tube as well as the middle tube. As a consequence, the water in the small basin is completely fresh and since it is well mixed by cooling from above, the dye is relatively uniform everywhere.

Regime number 3 is sketched in figure 4c. Salt water is found in the small basin. This regime is found for the greatest cooling rates, and it corresponds most closely to the 
"mixed-mode" described in section 2 and in figure 2. Clear salty water rapidly enters the small basin through the middle tube and ascends to the top. It gets cooled there and turbulently mixes with a very small amount of dyed fresh water that flows in through the top tube. The cooled mixture descends from top to bottom in the small basin as cold convection thermals, and the cold mixture leaves through the bottom tube.

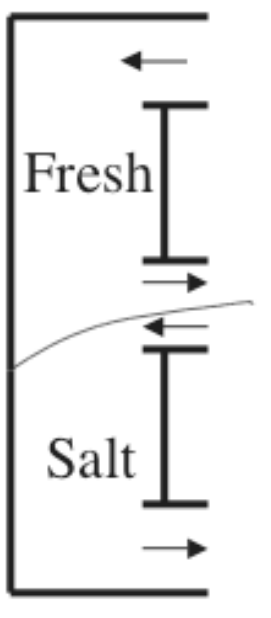

(a)

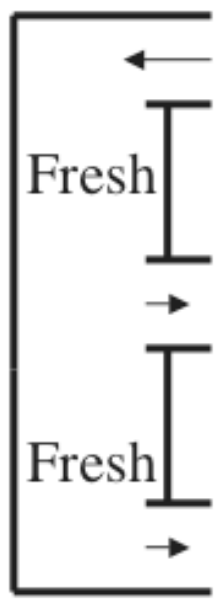

(b)

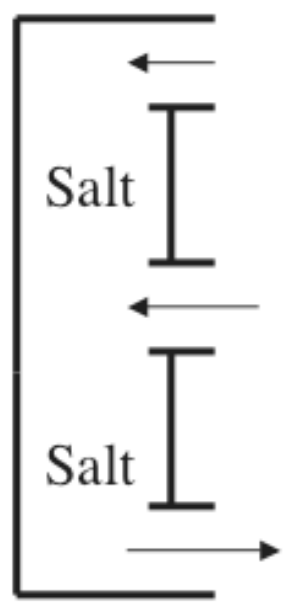

(c)

Figure 4. Flow direction through the tubes for the first three regimes of flow. a) Regime 1. b) Regime 2. c) Regime 3.

Regime number 4 has time-dependent flow. This regime involves layers in the small basin whose depth changes. Three photographs at different stages of the oscillation cycle are shown in Figure 5. As the layer of dyed fresh water is cooled in the small basin, the depth of the layer slowly increases and the color decreases by a small amount. Figure 5a shows such a layer after it has thickened and the interface has descended to the middle tube. Suddenly a new darker layer forms at the top. At the base of this new layer is a new internal interface that begins to move downward like the first interface (Figure $5 b)$. Below this new interface is the old layer, which is now a middle layer. Its color subsequently begins to fade dramatically. We presume that the source of fresh water to the middle layer has been cut off substantially by the formation of the new layer. The water in the bottom of the small basin begins to darken and we interpret this as showing increased transport of fresh water from the middle layer into the water at the bottom of the small basin. This implies that there is also increased transport of salt water from the water at the bottom of the small basin into the middle layer, which is consistent with the 
fading of color of the middle layer. In some of the cases, the interface of the middle layer is deeper than $D / 2$. In those cases, an exchange flow like that in regime 1 sets up in the middle tube and the interface depth subsequently changes very slowly or not at all. The inflowing salt water along the bottom of the middle tube may mix with the water and contribute to additional mixing of salt water into the middle layer. After some time interval, the interface below the middle layer begins to exhibit large vertical excursions and waves sometimes break. The large excursions become greater and more frequent with time. Flow into the small basin by clear salty water along the bottom of the middle tube (clear fluid in Figure 5b) seems to contribute to mixing as it descends through the lower layer. As time progresses, the interfacial waves in the lower interface break more frequently and the color difference between the lower layer and the fluid below it becomes much fainter. Suddenly (within about 5 minutes, as compared to a few hours for the entire cycle to happen) the bottom interface mixes away by direct overturning (Figure5c). The middle layer vanishes and only the upper interface is left (Figure 5c).
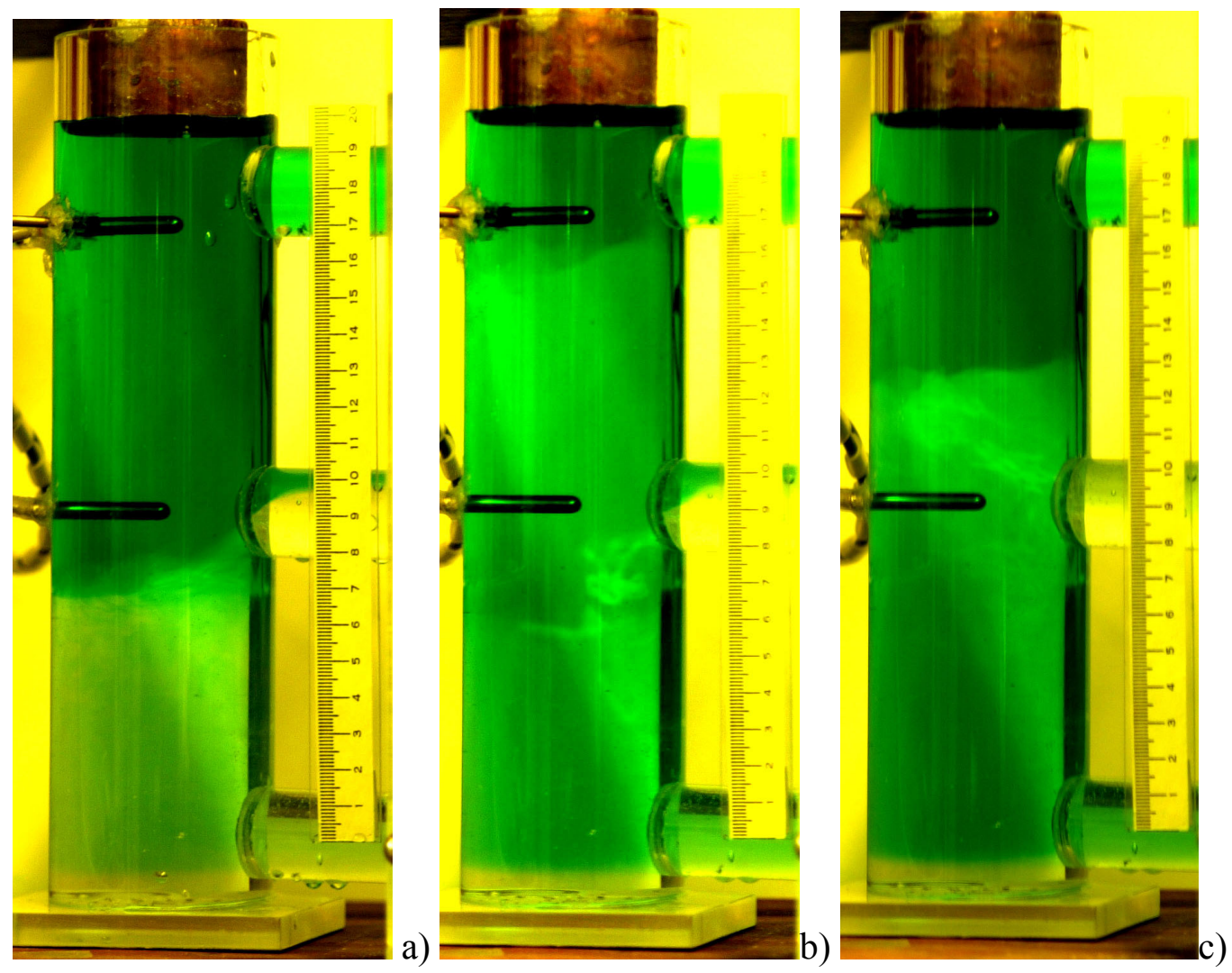

Figure 5. Photographs of a typical oscillation cycle. a) A layer of dyed freshwater has become thickened due to cooling from above. The interface has descended to the 
level of the middle tube. b) A new layer forms on top of the first layer. Note that a small amount of clear fluid is flowing into the small basin through the middle tube and descending through the lower layer. c) The lower layer has mixed away and the new layer is now left alone, and its interface is becoming deeper. The clear fluid is rising to the base of the new layer.

\subsection{Quantitative results}

The graph of $\tilde{T}$ and $\tilde{S}$ as functions of $\tilde{T} *$ in Figure 6 summarizes the experimental results and compares the points with a prediction from steady theory. To plot the predictions of theory for this experiment, the value of the two dimensionless numbers $\gamma$ and $\tilde{d}$ is required. The latter is easy to determine because it is simply a dimensionless number of the layer depth. This depth is determined by the depth of the drain. Its value is $\tilde{d}=0.05$. The dimensionless number $\gamma$ was found by conducting rundown experiments to calibrate the top and middle tubes. Dense water was drained through the tube to be calibrated under gravity and the time constant of the draining was calculated from the data. This was converted to a flow resistance coefficient for both the top tube and the middle tube. The ratio of the two coefficients is $\gamma=0.0094$. These two parameters were then used in a numerical model described in Whitehead (2000) to produce the predictions for dimensionless temperature (dashed) and salinity (solid) that are shown in figure 6.

These curves are compared to experimental data. To plot the experimental temperature data, the temperature is divided by $\beta S_{0} / \alpha$. The value of the coefficient of thermal expansion used is $\alpha=0.00021{ }^{0} C^{-1}$. This is the average value of expansion coefficient for water with the maximum salinity in the temperature range between $0^{\circ} \mathrm{C}$ and $40^{\circ} \mathrm{C}$. The average value for fresh water in the same temperature range has a coefficient of thermal expansion that is about $10 \%$ smaller. Naturally, due to nonlinearity in the equation of state of water, the coefficient of expansion in this temperature range varies enormously. It is zero at $4{ }^{0} \mathrm{C}$ for freshwater to almost twice $0.00021{ }^{0} \mathrm{C}^{-1}$ at $40{ }^{0} \mathrm{C}$. Therefore, the value we selected is somewhat arbitrary. Fortunately, it should give a measure of the driving forces that is good to within about 10\%. As mentioned above, the value of $\beta S_{0}$ is 0.0052 for all experimental runs. To plot the experimental 
salinity data, the difference of the densiometer reading from the density of fresh water is found, and it is divided by $\beta S_{0}=0.0052$.

These calibrations result in the curves and experimental data in figure 6. For runs that had steady mixed mode flows, the mean value of a salinity time series is shown as a plus and a mean of the temperature time series is shown as an asterisk. The time series records actually possess small time dependence that we attribute to convection cells. The standard deviation for both the temperature and salinity records for the mixed-mode is about as big as the asterisks. For steady fresh-mode runs the same symbols are enclosed in squares. Values with regime number one are plotted with the symbol $\mathrm{x}$ for both temperature and salinity. Convection is also present in the fresh-mode records and the regime number one records, and the standard deviation of these records are about as big as the asterisks.

The time-dependent oscillating runs have much greater standard deviations. The means are shown as solid circles for salinity, and open circles for temperature. Standard deviations are shown as error bars. The extreme value for each excursion of an oscillation is typically 1.5 to 2 times as big as the standard deviation. The periods are more than one hour for each cycle.

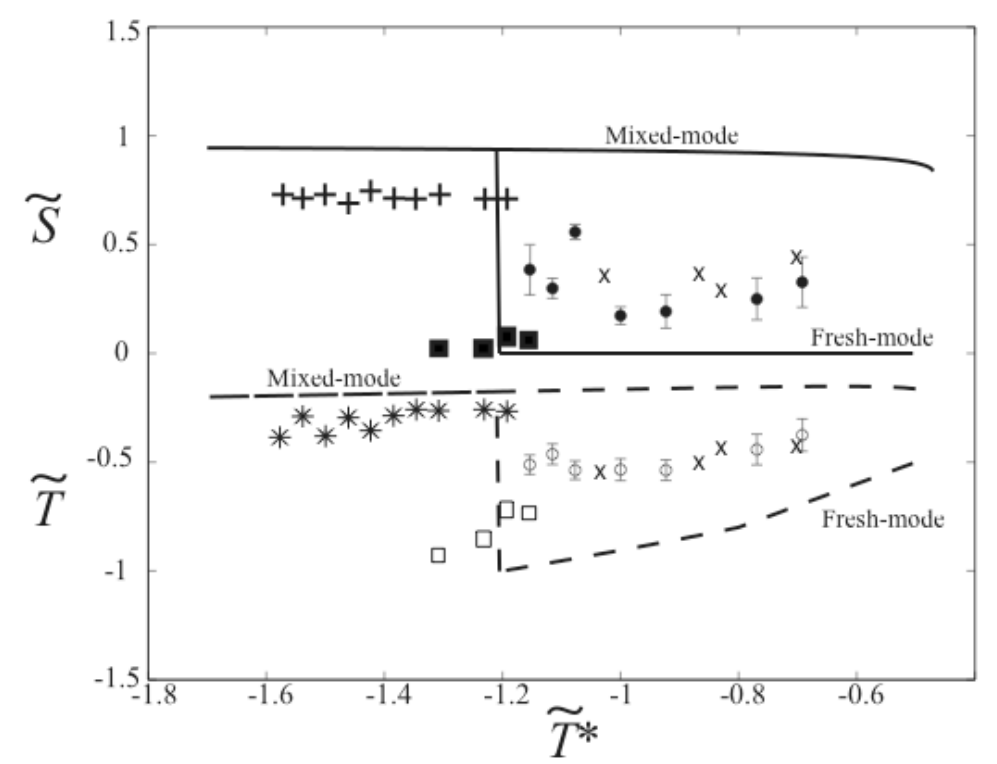

Figure 6. Dimensionless salinity and temperature (defined after equation (5)) as a function of $\tilde{T}^{*}$. The symbols are from experiments and the lines from theory. The solid line is salinity and the dashed line is temperature. The pluses are values of salinity and the stars are corresponding 
temperature, both averaged over a number of hours and for regime 3 (the mixed-mode). Solid squares are regime 2 (the fresh mode) average salinity and open squares are corresponding average temperature. Solid circles are regime 4 (oscillations) average salinity and the error bars give standard deviations. Open circles give corresponding values for temperature. The x's give values for regime 1.

Here is a verbal summary of what appears to happen during an oscillation cycle. Qualitatively, the cycle is completely obvious when viewing the videos. These motivated the sequence of photographs in figure 5. Quantitatively, figure 7 shows temperature and salinity data for over two cycles. As the thin layer cools, it becomes denser. This produces an inflow of fresh water through the top tube so that the bottom interface of the layer in the small basin descends and passes the temperature probes. Simultaneously, the cold temperature of the layer in the small basin causes a heat flux into the bottom of the layer from the warmer water below it. This produces double-diffusive transport of heat and salinity up into the layer. The temperature of the layer rapidly adjusts to a balance between heating from below and cooling from above. We believe, but have not measured, that flow of fresh water into the layer through the top tube becomes very small after this adjustment. In any event, the top layer gradually becomes saltier with time. Ultimately, the layer becomes salty enough and cold enough to achieve the same density as the deep water. When this happens, the interface overturns and the layer mixes away. Around this time the cycle is repeated with the initiation of a new thin layer of pure fresh water. This layer frequently starts before overturn as in figure 7 , but there are cases where the new layer appeared to be simultaneous with overturn. Periods ranged from about two to ten hours for the assorted runs. 


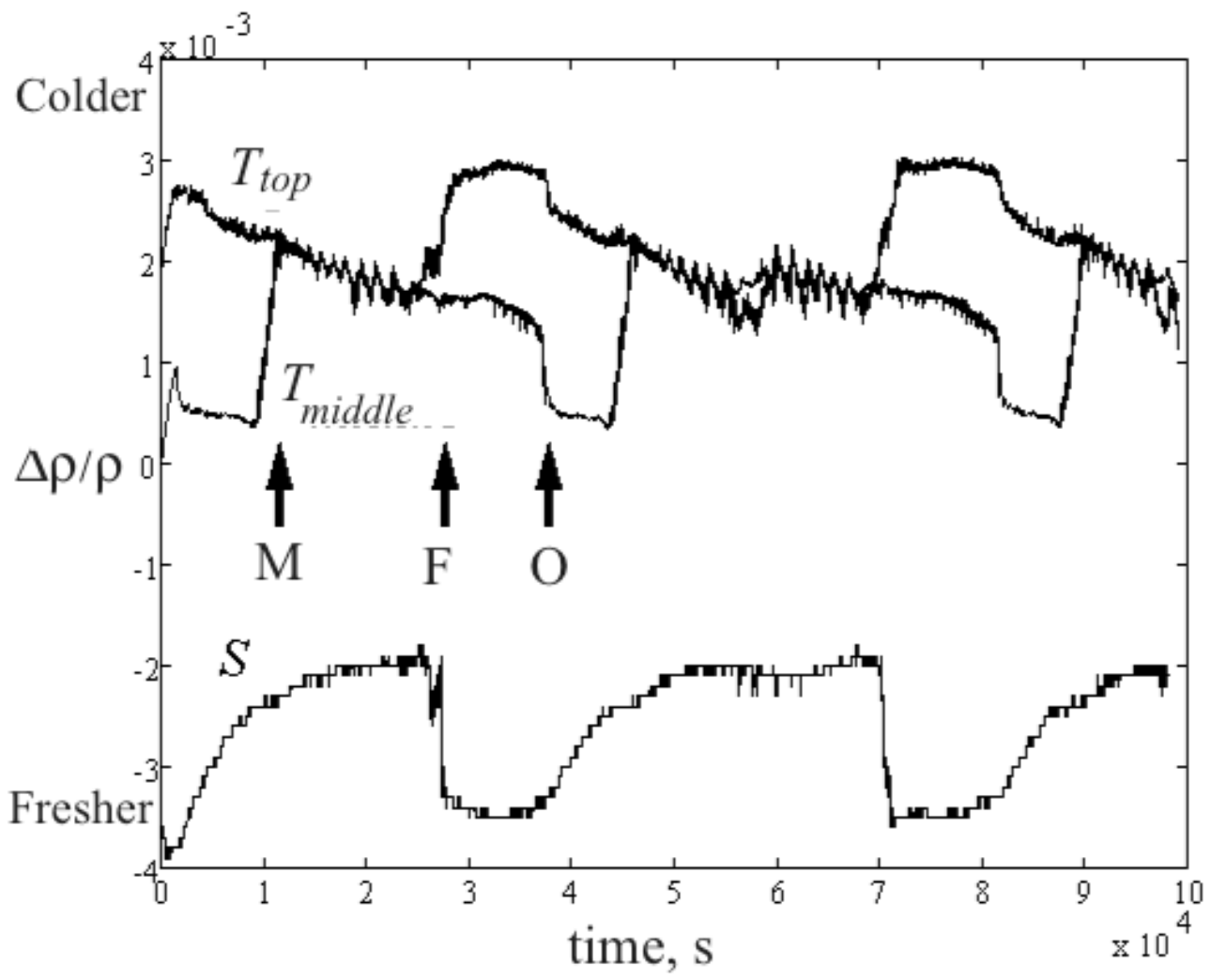

Figure 7. Density variation during three oscillation cycles. The forcing strength is $\alpha \Delta T / \beta S_{0}=0.5$. The top two curves show values of $-\alpha T$ at $0.025 \mathrm{~m}$ depth and at $0.1 \mathrm{~m}$ depths (labeled " $T_{\text {top }}$ " and " $T_{\text {middle }}$ ") respectively. Density change from $S_{0}$ subtracted from the density from the water pumped to the densiometer from $2.5 \mathrm{~cm}$ depth of the small basin $\beta\left[S_{0}-S\right]$ is also plotted here and labeled by the "S". The letter "M" corresponds to the time when the layer is seen on videotape to reach the middle thermistor. The letter " $F$ " shows the time when a new layer that has formed is seen to reach $2.5 \mathrm{~cm}$ depth. The letter "O" shows when the middle layer is seen to overturn.

\section{Discussion}

The experiment exhibited two ranges with apparent multiple equilibrium. The range of the driving parameter $\tilde{T} *$ that both modes occupy and the range of multiple equilibria for both modes is considerably smaller than theory. The same two facts, that the jumps are found but that the range of multiple equilibria is reduced, is also reported 
for other T-S experiments with more degrees of freedom than comparable box models (Whitehead et al. 2003).

Only four runs had regime number 1 and so this regime might be regarded as rather poorly observed with the experiments to date. However, we were careful to run each experiment long enough to be convinced that it had no drift so that it was not a slow part of an oscillation. Thus, each point with regime 1 is recorded with at least 12 hours of steady data. In addition, we had also seen regime 1 in five runs of exploratory experiments before arriving at the present experiment. (We had conducted three such exploratory experiments, two sets are reported in Te Raa 2001, and another set is a prototype used the present apparatus, but did not record density). Thus regime 1 seems to be robust, Because of this, we believe that the regime is stable and completely reproducible. It is clearly found near the same forcing parameters as the oscillations, so possibly it is meta-stable.

The oscillation seems to be new, and a complete report describing the oscillation and presenting models of it is in preparation. Experiments by Te Raa (2001) were the first that documented oscillations like these. The apparatus was an upside-down version of the present ones with a shallow layer of salt water on the bottom along with the small basin heated from below rather than cooled from above. There was rough agreement (within tens of percent) between theory and experiment for the temperature of the layer and the transition value of temperature. Tozuka (2002) conducted a numerical study of a layered numerical model like the one sketched in figure 1, but with a parameterized mixing between the fresh water layer and the layer below it. The interface entrainment was parameterized using a "Krauss-Turner" relation for interfacial mixing that is more appropriate for the mixed layer of the ocean rather than the laboratory experiment. He found that the model produces two distinct oscillations. When a new layer forms, a complex balance near the top complicates the oscillating cycle. The inflowing fresh water encounters thermal convection, and the competition either mixes up the fresh water or instead it starts a new layer before overturning happens. This along with other details of the cycles are discussed in the work in preparation.

If interfacial mixing at the base of the fresh water layer gradually causes the layer to increase in salinity as found over long times in this experiment, then there may be 
some similarities between this experiment and ocean cycles. We wonder whether in the ocean the salinity response would follow the thermal adjustment time-scale multiplied by some function of the density ratio. There are not yet observations of either multiple equilibrium or self-driven oscillations in the ocean or in bays, but rapid climate change is found in numerous ocean models. In addition, the role of the ocean in climate fluctuations is generally accepted now (Weart 2003). In any case, these laboratory studies show the oscillations in some detail so that we know they can actually exist and we present a plausible mechanism for their dynamics.

\section{Summary}

The experiment exhibited the two different flow states predicted by theory (Whitehead 2000), namely the mixed-mode (regime 3) and the fresh-mode (regime 2). The distinct difference in temperature and salinity between the two modes is clearly verified in the experiment (Figure 6). However, the range of the driving parameter $\tilde{T}^{*}$ that both modes occupy and the range of multiple equilibria for both modes is considerably smaller than theory. This is also reported for other T-S experiments with more degrees of freedom than comparable box models (Whitehead et al. 2003). For $\tilde{T} *$ warmer than -1.15 , regime 1 and regime 4 replace the fresh and mixed modes. The two regimes exhibit values of $\tilde{T}$ and $\tilde{S}$ that fall along the same trend. This trend has values that lie between the values of the mixed and fresh modes in the theory. Regime 1 is absent in the theory of Whitehead (2000) because it involves exchange flow in the middle tube, which was not included in the theory. Regime 4 is absent from that theory because it is oscillatory and it appears to involve the transport of heat and salinity through the bottom of the layer, which was not included in the theory. More aspects of the oscillation are to be found in a companion report that is under preparation in conjunction with other colleagues.

\section{Acknowledgements}

The National Science Foundation, Physical Oceanography Section under Grant OCE-0081179 supported the experimental laboratory studies. The Paul M. Fye Chair of the Woods Hole Oceanographic Institution provided support for part of the laboratory 
work of JW. The help of Jeanne Fleming is gratefully acknowledged, and Kaylee Lino helped with photography. Woods Hole Oceanographic Institution Contribution \#10962.

\section{References}

Bryan, F. (1986) High-Latitude salinity effects and interhemispheric thermohaline circulations. Nature 323, 301-304 1986.

Gordon, A. L., (1991) Two stable modes of Southern Ocean Winter Stratification. In: Deep Convection and Deep Water Formation in the Oceans, Netherlands, P.C. Chu and J.C. Gascard, Editors, Elsevier Science Publishers; p. 17-35.

Hearn, C. J. and H. S. Sidhu (1999) The Stommel model of shallow coastal basins. Proceedings of the Royal Society of London, A455, 3997-4011.

Lab Sea Group (1998) The Labrador Sea deep convection experiment. Bulletin of the American Meteorological Society, 10, 2033-2058.

Manabe, S., and Stouffer, R. J. (1995) Simulation of abrupt climate change induced by freshwater input to the North Atlantic Ocean. Nature, 378, 165-167.

Marotzke J. (1994) Ocean Models in Climate Problems. In Ocean Processes in Climate Dynamics: Global and Mediterranean Examples ed. P. Malanotte-Rizzole and A.R. Robinson, 79-109. Dordrecht: Kluwer Academic Publishers.

Medoc Group (1969). Observations of formation of deep water in the Mediterranean Sea, Nature, 227, 1037-1040.

Rahmstorf, R. and A. Ganopolski (1999) J. Climate, 12, 1349-1352.

Stommel H. (1961) Thermohaline convection with two stable regimes of flow. Tellus, $13,224-30$.

Stommel, H. (1962). On the smallness of sinking regions in the ocean. Proceedings National Academy of Sciences, 48, 766-772

Sverdrup, H.U., M.W. Johnson and R.H. Fleming (1942) The Oceans: their Physics, Chemistry and General Biology, Prentice-Hall, 1087 pp.

Te Raa, L. (2001): Convective oscillations in a laboratory model. GFD Fellow Report, http://gfd.whoi.edu/proceedings/2001/PDFvol2001.html. 
Tozuka, Tomoki (2002) On cyclic and oscillatory convection in a simplified box model with entrainment. http://gfd.whoi.edu/proceedings/2002/PDFvol2002.html.

Weart, Spencer (2003) The discovery of Rapid Climate Change, Physics Today, 56, \#8, 30-36.

Whitehead, J.A. (1995) Thermohaline ocean processes and models. Annual Review Fluid of Mechanics, 27, 89-113.

Whitehead, J. A. (1996) Multiple states in doubly driven flow. Physica D, 97, 311-321.

Whitehead, J. A. (1998) Multiple T-S states for estuaries, shelves and marginal seas. Estuaries, 21, 278-290.

Whitehead, J. A. (2000) Stratified convection with multiple states. Ocean Modelling, 2, 109-121.

Whitehead, J. A. , M. L. E. Timmermans, W. Gregory Lawson, S. N. Bulgakov, A. M. Zatarian, J. F. A. Medina, and John Salzig (2003) Laboratory studies of thermally and/or Salinity-driven flows with partial mixing: Part 1 Stommel transitions and multiple flow states, J. Geophys. Res. 108, No. C2, 3036, doi:10.1029/2001JC000902. 